\title{
Investigation of Phase Pattern Modulation for Digital Fringe Projection Profilometry
}

\author{
Cheng-Yang Liu, Chung-Yi Wang \\ Department of Biomedical Engineering, National Yang-Ming University, Taipei City, Taiwan, cyliu66@ym.edu.tw
}

\begin{abstract}
The fringe projection profilometry with sinusoidal patterns based on phase-shifting algorithms is commonly distorted by the nonlinear intensity response of commercial projector. In order to solve this issue, sinusoidal width modulation is presented to generate binary sinusoidal patterns for defocusing the projection. However, the residual errors in the phase maps are usually notable for highly accurate threedimensional shape measurements. In this paper, we propose the fringe patterns of the sinusoidal, square, and triangular periodic waveforms with seven-step phase-shifting algorithm to further improve the accuracy of three-dimensional profile reconstruction. The absolute phase values are calculated by using quality guided path unwrapping. We learn that by properly selecting fringe patterns according to the target shape, the undesired harmonics of the measured surface have negligible effect on the phase values. The experiments are presented to verify the imaging performances of three fringe patterns for different testing targets. The triangular fringe patterns are suitable for the shape measurements of complex targets with curved surfaces. The results provide a great possibility for high-accuracy shape measurement technique with wider measuring depth range.
\end{abstract}

Keywords: Digital imaging, fringe projection, waveform.

\section{INTRODUCTION}

Recently, optical measurement has been an indispensable technique for many applications such as manufacturing process, virtual reality, and biomedical engineering [1]-[3]. General optical three-dimensional (3D) shape measurements are based on structured light illumination and phase calculation [4]. According to the analytical approach of the phase distributions, these measurements are divided into two ways: spatial domain method and transform domain method. The spatial domain method usually employs phase-shifting calculation to operate the primary data, for example, the common fringe projection profilometry (FPP) [5]. The FPP is a non-contact, non-interferometric, and high-speed technique which has been used extensively for profiling 3D complicated surfaces [6]-[12]. In the FPP, the structured illumination with typical sinusoidal fringe projected onto the target surface will get distorted pattern because of the surface height variations [13]-[15]. The distorted fringe patterns with spatial variations involve the topological information of the targets [16]-[18]. The digitized full-field information from the region of interest is easily acquired by using scalable FPP. The FPP is a versatile inspecting technique which can be adapted for space-constrained assembling and less-than-ideal illumination situations [19]-[22]. These imaging applications usually demand a qualitative detection and quality-dense topological information from the targets. Several algorithms for frequency estimation of fringe pattern have been presented in previous references [23]-[31]. These algorithms are usually based on spatial-frequency methods such as windowed Fourier and wavelet transforms. The local frequency estimation can be achieved by these algorithms, but the imaging resolution is limited by the sinusoidal patterns. Furthermore, the peak location search calculation involved in these algorithms requires long computational time.

3D shape measurement based on FPP can be easily achieved by using a commercial video projector. However, an off-the-shelf projector has the nonlinear response of the projection engines. The response calibration of a projector is usually required for high-quality $3 \mathrm{D}$ shape measurement. Unfortunately, the nonlinear response cannot be eliminated by one-time calibration because the projected intensity response actually varies with the time lapse. In order to solve this problem, the methods of binary sinusoidal fringe patterns are proposed to avoid the nonlinearity effect [8], [10], [12]. The quality of $3 \mathrm{D}$ shape measurement is improved by encoding the binary fringe patterns in three-color channels. Nevertheless, these encoding methods reduce the measurement speed and the measuring depth range is limited by coding errors from the out-of-focus blur. In this study, the fringe patterns of the sinusoidal, square, and triangular periodic waveforms with seven-step phase-shifting algorithm are proposed to further improve the accuracy of $3 \mathrm{D}$ profile reconstruction. The dominant error-causing harmonics of the 
measured surface can be reduced by properly selecting fringe patterns. The experiments are performed to verify the imaging quality of three fringe patterns for different testing targets. The description of the method is presented in Section 2. The experimental results and discussion of the testing targets are illustrated in Section 3. The conclusion is presented in Section 4.

\section{DESCRIPTION OF THE METHOD}

The FPP-based technique is employed for detecting the 3D shape of the testing targets. Fig.1. shows the experimental arrangement. A DLP projector (Acer $\mathrm{K} 132+$ ) is used for projecting different fringe patterns on the target surface. A high-quality CCD camera (JAI AT-200CL) is used for capturing subsequent fringe patterns from the target surface. Fig.1.a) depicts a crossed-optical-axes triangulation which is usually applied in the digital FPP. The CCD camera is mounted on a motorized rotating arm (Sigma Koki KST$160 \mathrm{YAW}$ ) so that the detecting angle could be varied precisely. Fig.2.a) shows the sinusoidal fringe pattern. In general, sinusoidal fringe distribution for $\mathrm{N}$-step phaseshifting algorithm with equal phase shifts can be characterized as:

$$
\mathrm{I}_{n}(x, y)=\mathrm{A}(x, \mathrm{y})+\mathrm{M}(x, y) \times \cos [\Phi(x, y)+2 \pi n / \mathrm{N}]
$$

where $x$ and $y$ are the pixel indices along vertical and horizontal axes in the fringe pattern, $\mathrm{A}(x, \mathrm{y})$ is the average intensity of illumination, $\mathrm{M}(x, y)$ is the intensity modulation of fringe pattern, $n$ is phase-shift index, and $n=1,2,3, \ldots, \mathrm{N}$. $\Phi(x, y)$ is the phase to be solved by the following equation:

$$
\Phi(x, y)=\tan ^{-1} \frac{\sum_{n=1}^{\mathrm{N}} \mathrm{I}_{n}(x, y) \sin (2 \pi n / \mathrm{N})}{\sum_{n=1}^{\mathrm{N}} \mathrm{I}_{n}(x, y) \cos (2 \pi n / \mathrm{N})}
$$

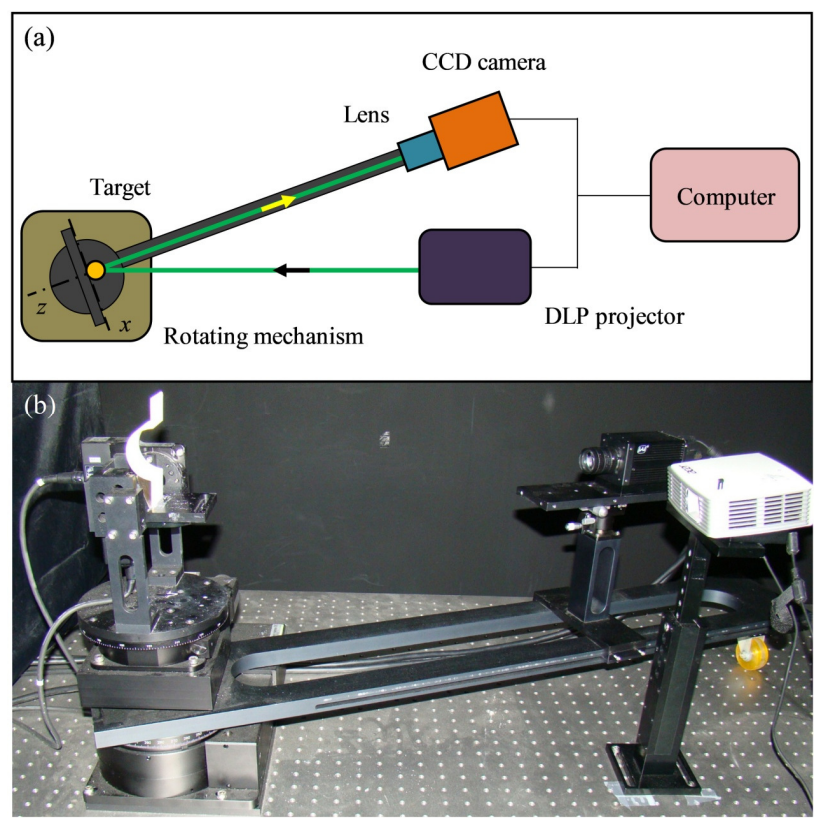

Fig.1. Digital fringe projection profilometry: a) schematic diagram and b) photograph.
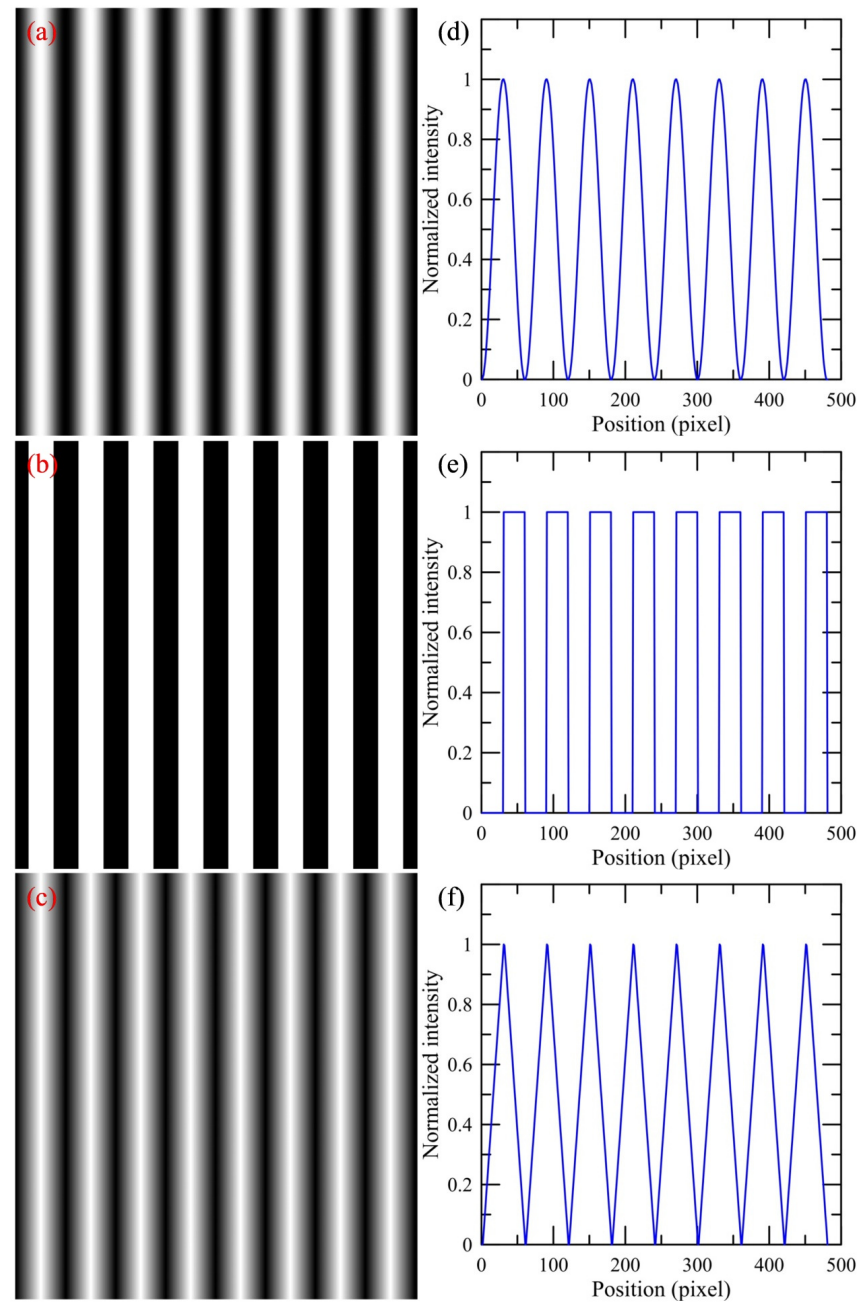

Fig.2. Projective fringe patterns with a) sinusoidal, b) square, and c) triangular periodic waveforms. Cross-sections of d) sinusoidal, e) square, and f) triangular patterns.

The average intensity of illumination and the intensity modulation of fringe pattern are assumed to be the same value in theory. Fig.2.d) shows the cross-section of sinusoidal pattern with a constant intensity. This fringe pattern is a simple continuous sinusoidal signal and is defined within a multiple of $2 \pi$ period. However, the sinusoidal signal has a finite range and the curve of the signal is slightly deformed in the experiment. The $\mathrm{A}(x, \mathrm{y})$ and $\mathrm{M}(x, y)$ may fluctuate during phase-shifting experiments. These fluctuations cause phase errors in the traditional phase-shifting calculations [32], [33]. In the fringe patterns, we usually process 8-bit gray-level images with intensity levels from 0 to 255 at each pixel. A gray-level image can be represented as the super-position of time-multiplexed binary patterns $\mathrm{M}_{i}$ with $i=1,2,3, \ldots, 8$. In these binary patterns, the most critical pattern of the binary representation is $M_{1}$. Fig.2.b) shows the binary fringe pattern with square periodic waveform. The binary (square) fringe distribution for each gray-level image can be reconstructed through an equation as:

$$
\mathrm{I}_{n}(x, y)=\sum_{i=1}^{8} 2^{(8-i)} \mathrm{M}_{i n}(x, y)
$$


where $\mathrm{M}_{i 1}$ is the sequence of the binary fringe patterns corresponding to $n=1$. Fig.2.e) shows the cross-section of binary fringe pattern with square periodic waveform. The intensity of the binary fringe pattern takes the values 0 or 1 depending on the integer number of the position. Because the binary fringe patterns only contain zeros and ones, it is possible to eliminate the nonlinear response of the projector and camera. Furthermore, we also propose the triangular fringe patterns as a way to solve the problems caused by gamma nonlinearity in FPP. Fig.2.c) shows the triangular fringe pattern. The triangular fringe distribution for a sequence of phase-shifted patterns can be described as:

$$
\mathrm{I}_{n}(\mathrm{x}, \mathrm{y})= \begin{cases}\frac{2 \mathrm{M}(x, y)}{\mathrm{T}}\left(x+\delta_{n}\right)+\frac{\mathrm{M}(x, y)}{2} & x+\delta_{n} \in\left[0, \frac{\mathrm{T}}{4}\right) \\ \frac{2 \mathrm{M}(x, y)}{\mathrm{T}}\left(x+\delta_{n}\right)+\frac{3 \mathrm{M}(x, y)}{2} & x+\delta_{n} \in\left[\frac{\mathrm{T}}{4}, \frac{3 \mathrm{~T}}{4}\right) \\ \frac{2 \mathrm{M}(x, y)}{\mathrm{T}}\left(x+\delta_{n}\right)-\frac{3 \mathrm{M}(x, y)}{2} & x+\delta_{n} \in\left[\frac{3 \mathrm{~T}}{4}, \mathrm{~T}\right)\end{cases}
$$

where $\mathrm{T}$ is the pitch of the fringe pattern and $\delta_{n}$ is the $n$th phase-shifting distance along the $x$-axis, which is defined by:

$$
\delta_{n}=(n-1) \frac{\mathrm{T}}{\mathrm{N}}, n=1,2, \ldots, \mathrm{N}, \mathrm{N} \geq 2
$$

Binary-code fringe patterns only comprise black and white pixel values and the pattern decoding is relatively simple. Fig.2.f) shows the cross-section of triangular fringe pattern with gray-codes. Binary reflected gray-codes are also suitable for binary encoding because the pixel value only changes at a time. We propose to project and acquire a sequence of strictly binary fringe patterns for synthesizing a high-quality triangular fringe pattern. The $\mathrm{M}_{i}$ receive either value 1 or 0 depending on the integer number of $\mathrm{I}_{n}(x, y)$. Normal fringe pattern is represented by a pure binary-code pattern which is commonly utilized in image processing. Thus, the triangular fringe pattern sequence is projected on the surface of testing targets. The wrapped phase values are calculated by applying seven-step phase-shifting calculation in equation (2). The differential phase angle between two following fringes is in multiples of $2 \pi$ and the wrapped phase values are in a module of $2 \pi$. The quality guided path calculation is used to demodulate wrapped phase values with high reliability [34]. The phase-unwrapping calculation could also eliminate the phase errors caused by discontinuous surfaces. The unwrapping path is determined by the pixel reliability and the absolute phase values are obtained along a discontinuous route. The relationship between absolute phase value and real dimension is quantified by the triangulation method [35]. Because the imaging intensity of deformed fringe patterns from the target surface is a key parameter for phase-shifting calculation, our digital FPP is placed inside a darkroom to prevent any influence of ambient light in the environment.

\section{EXPERIMENTAL RESULTS AND DISCUSSION}

The validation experiments have been performed to verify the performance of different fringe projections. The sinusoidal, square, and triangular fringe patterns are generated by a DLP projector with a resolution of $1024 \times 768$. The distorted fringe pattern images are captured by using a
3 CCD camera with a resolution of $1620 \times 1236$ under a viewing angle of $20^{\circ}$. The projector and camera are calibrated by means of a reference plane marked by black and white grids. Fig.3.a) to Fig.3.c) depict three testing targets of semicircular, rectangular, and triangular shapes. The nominal diameter of the semicircular testing target is $10 \mathrm{~mm}$. The nominal width and height of the rectangular target are $10 \mathrm{~mm}$ and $10 \mathrm{~mm}$. The nominal base-side and height of the isosceles triangular target are $10 \mathrm{~mm}$ and $10 \mathrm{~mm}$. The material of these testing targets is polylactide (PLA) and they are made by using a commercial 3D printer. Fig.3.d) to Fig.3.f) depict one triangular fringe pattern projected on different testing target surfaces. The fringe patterns are deformed with respect to the target surface. A total of seven fringe patterns are recorded into an industrial computer for post-processing. The fringe pitch is $1 \mathrm{~mm}$ and the phase shift between each fringe pattern is $\pi / 2$. The seven-step phase-shifting calculation is performed on these distorted fringe patterns [16].
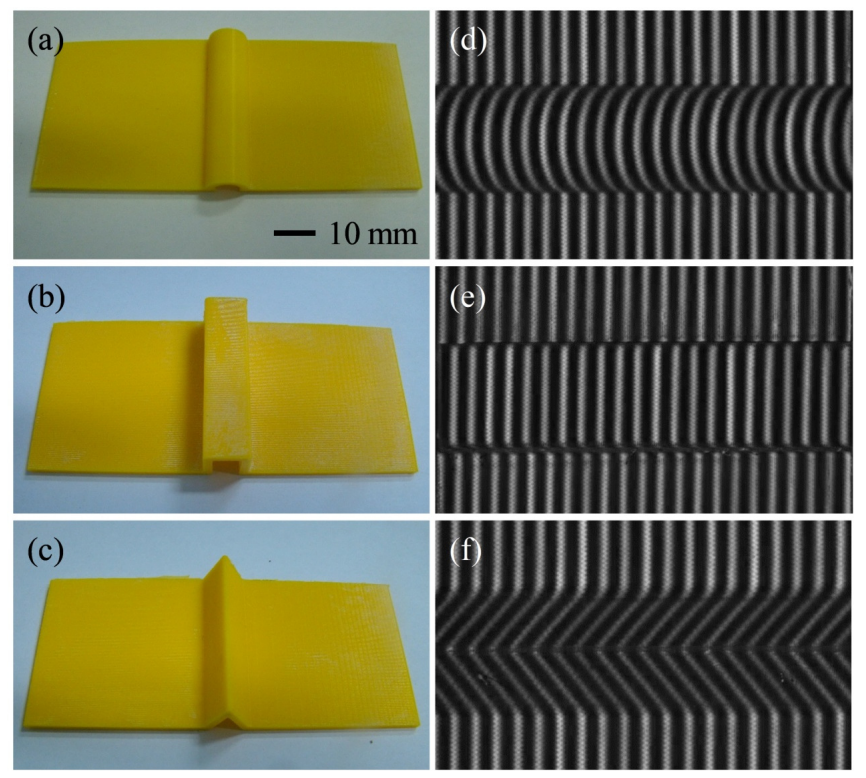

Fig.3. Testing targets of a) semicircular, b) rectangular, and c) triangular shapes. Digital fringe patterns projected on the d) semicircle, e) rectangular, and f) triangular target surfaces.

Fig.4. depicts the wrapped phase maps calculated by sevenstep phase-shifting algorithm with sinusoidal, square, and triangular fringe patterns for three testing targets. It is shown that the modification in fringe pattern especially affects the wrapped phase map. The red arrows in Fig.4. indicate the phase errors in the wrapped phase maps. The appearance of phase errors depends on the projected fringe patterns. From these wrapped phase maps, we observe that the phase errors corresponding to bright fringes become inconspicuous for the triangular fringe projection. In Fig.4., the wrapped phase values are a periodic function of $2 \pi$. The quality guided path calculation is applied to unwrap these phase values. Fig.5. illustrates the unwrapped phase maps calculated by quality guided path algorithm with sinusoidal, square, and triangular fringe patterns for three testing targets. The values of unwrapped phase are a continuous rise in the number in which 
white indicates the highest value and black indicates the lowest value. The rising trend of unwrapped phase value for sinusoidal, square, and triangular fringe projections can be observed in Fig.5. The unwrapped phase map distinctly indicates target features. Although phase error propagation can be exterminated by using quality guided path calculation, we still observe small ripple-like artifacts induced by the square fringe projection.

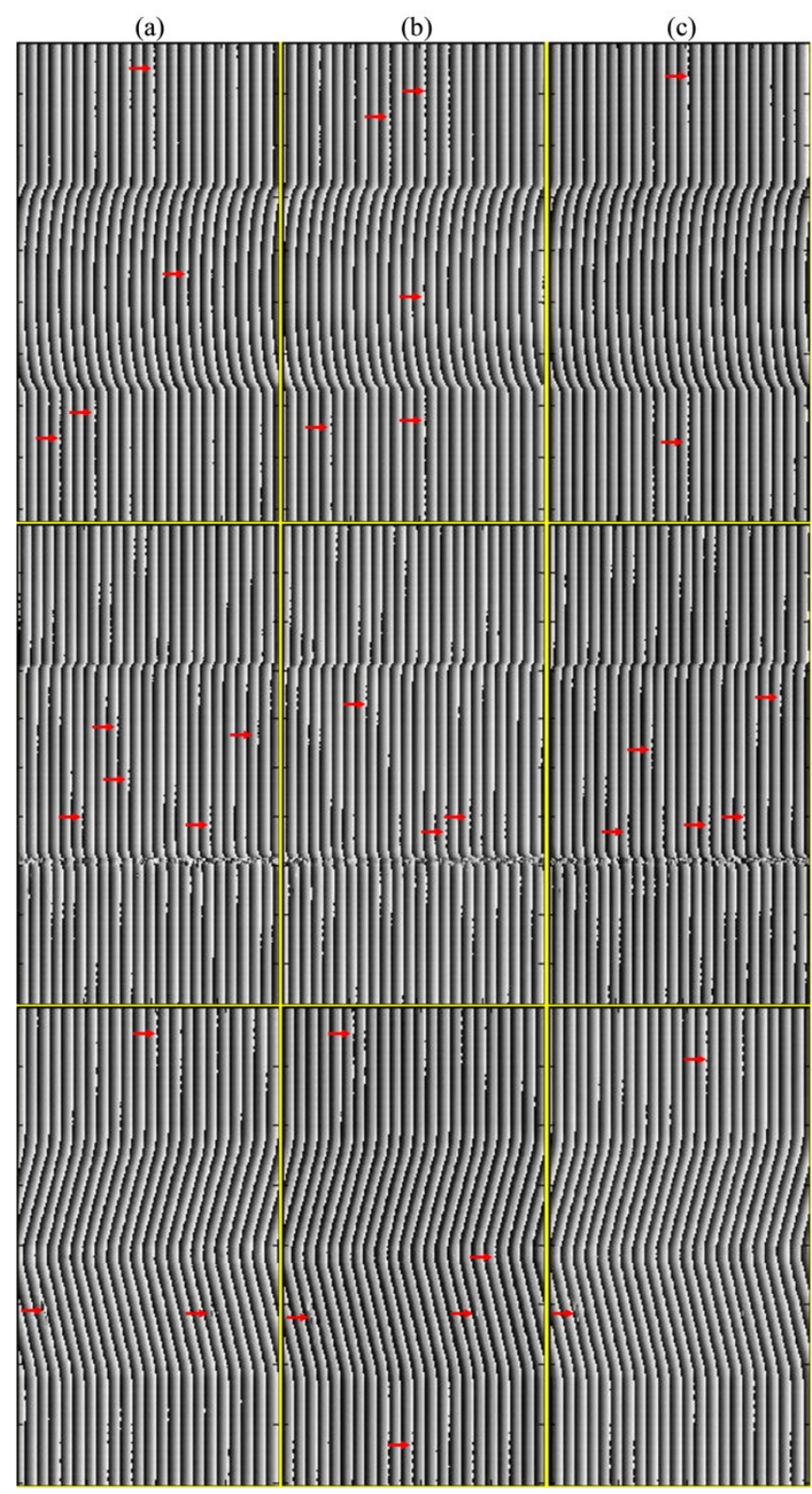

Fig.4. Wrapped phase maps calculated by seven-step phase-shifting algorithm with a) sinusoidal, b) square, and c) triangular fringe patterns for the semicircular (the first row), rectangular (the second row), and triangular (the third row) testing targets. The red arrows indicate the phase errors in the wrapped phase maps.

The background perturbation in the unwrapped phase maps can be removed by using the reference plane reduction method [36]. The absolute phase values without background noise are obtained by subtracting unwrapped phase values of a reference plane. The real $3 \mathrm{D}$ reconstructions of testing targets are acquired by calibrating the relationship between absolute phase values and real dimensions. Fig.6. depicts the $3 \mathrm{D}$ reconstructions of the semicircular, rectangular, and triangular testing targets with sinusoidal, square, and triangular fringe pattern projections. 3D surface shapes of the testing targets can be reproduced by the proposed fringe patterns. These experiments clearly show that either sinusoidal or triangular fringe projections could contribute reasonable results for the semicircular testing target, despite the small improvement that the triangular FPP has over the sinusoidal FPP. The measurement results with clearly noticeable ripples are produced for the rectangular testing target, specially projected by the square fringe pattern. The sinusoidal and triangular fringe projections could generate good 3D representations for the triangular testing target, but triangular FPP is slightly better than sinusoidal FPP, as shown in Fig.6.

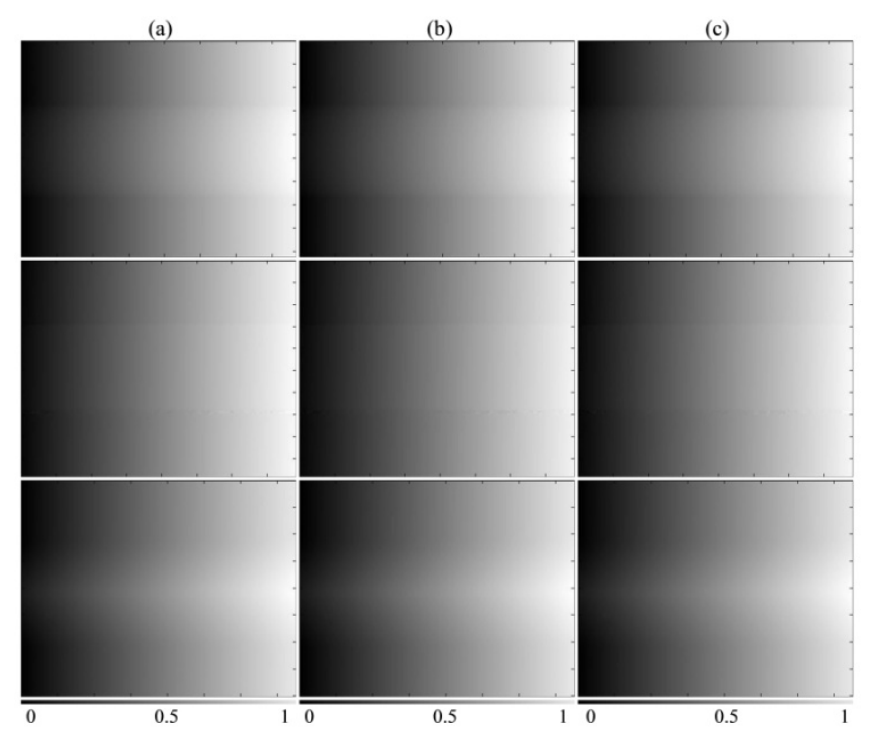

Fig.5. Unwrapped phase maps calculated by quality guided path algorithm with a) sinusoidal, b) square, and c) triangular fringe patterns for the semicircular (the first row), rectangular (the second row), and triangular (the third row) testing targets.

In order to compare the imaging differences between sinusoidal, square, and triangular FPP, the cross-section profiles of three testing targets by different fringe pattern projections are illustrated in Fig.7. The location of these cross-section profiles is at the center of a $3 \mathrm{D}$ representation of a measured target. The shape deformation in square FPP is larger than in sinusoidal and triangular FPP. In Fig.7.a), we can see that sinusoidal FPP produces low-frequency corrugations of the top measured surface. In Fig.7.c), triangular FPP creates the significant profile in which the surface ripples are hardly noticeable. The triangular FPP obviously mended the smoothness of the target surface. 
(a)

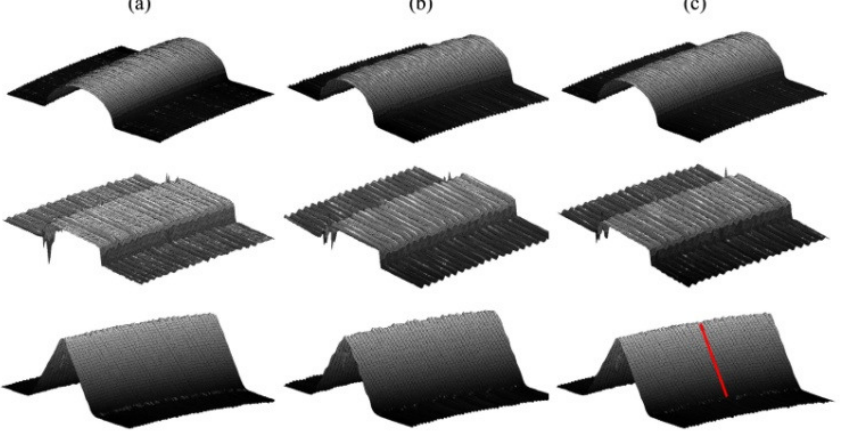

Fig.6. 3D representation of the semicircular (the first row), rectangular (the second row), and triangular (the third row) testing targets with a) sinusoidal, b) square, and c) triangular fringe pattern projections. The red line in the bottom right indicates the location of the cross-section profile.
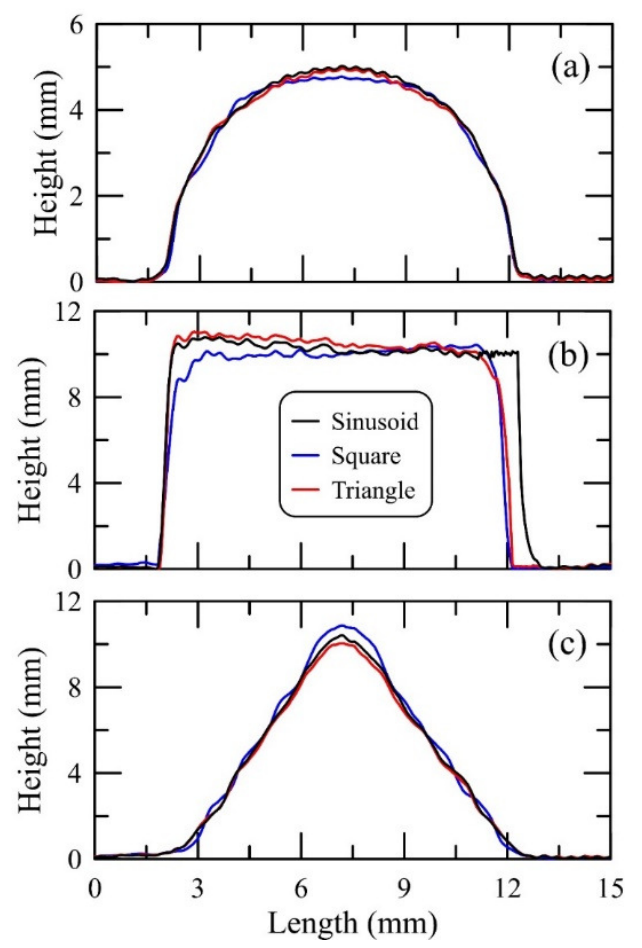

Fig.7. Cross-section profiles of the a) semicircular, b) rectangular, and c) triangular testing targets with different fringe pattern projections.

In order to quantify the total imaging errors by different waveforms, the total imaging error is defined as $\mathrm{R}=\left(\mathrm{A}_{m}-\mathrm{A}_{s}\right)$ / $\mathrm{A}_{s}$, where $\mathrm{A}_{m}$ is the measured area of the target profile and $\mathrm{A}_{s}$ is the standard area of the target profile. Table 1. shows the total imaging errors calculated by measured area and standard area. This calculation indicates that triangular FPP for curved surface of targets can outperform sinusoidal FPP. The tolerances of measured diameter of semicircular target are $0.11 \mathrm{~mm}$ for sinusoidal FPP, $0.27 \mathrm{~mm}$ for square FPP, and $0.06 \mathrm{~mm}$ for triangular FPP. The tolerances of measured height of rectangular target are $1.36 \mathrm{~mm}$ for sinusoidal FPP, $0.71 \mathrm{~mm}$ for square FPP, and $1.05 \mathrm{~mm}$ for triangular FPP. The tolerances of measured height of isosceles triangular target are $0.31 \mathrm{~mm}$ for sinusoidal FPP, $0.86 \mathrm{~mm}$ for square FPP, and $0.18 \mathrm{~mm}$ for triangular FPP. In other words, the triangular FPP is already good enough if narrow fringe patterns are used for complex targets with curved surfaces. On the other hand, the square FPP is suitable for target measurement with flat surface. These experiments indicate that the triangular FPP shall be accepted for high-quality 3D shape measurement. In the future work, the measurement uncertainty analysis of the FPP system will be presented for improving the accuracy of the triangular phase-shifting calculation.

Table 1. Total imaging errors calculated by measured area and standard area.

\begin{tabular}{|l|c|c|c|}
\hline & $\begin{array}{c}\text { Sinusoidal } \\
\text { FPP }\end{array}$ & $\begin{array}{c}\text { Square } \\
\text { FPP }\end{array}$ & $\begin{array}{c}\text { Triangular } \\
\text { FPP }\end{array}$ \\
\hline $\begin{array}{l}\text { Semicircular } \\
\text { target }\end{array}$ & $3.8 \%$ & $6.3 \%$ & $3.7 \%$ \\
\hline $\begin{array}{l}\text { Rectangular } \\
\text { target }\end{array}$ & $7.8 \%$ & $5.8 \%$ & $7.5 \%$ \\
\hline Triangular target & $5.8 \%$ & $5.9 \%$ & $5.1 \%$ \\
\hline
\end{tabular}

\section{CONCLUSION}

This paper presented the digital phase pattern modulation for $3 \mathrm{D}$ shape measurements based on the multiple FPP to overcome the problems associated with traditional fringe projection techniques. Three types of testing targets are employed to estimate imaging performance for different fringe projections. The fringe patterns of the sinusoidal, square, and triangular periodic waveforms with seven-step phase-shifting algorithm are performed to reconstruct the 3D surface profile of the testing targets. We have analyzed the imaging shape differences and total imaging errors of different fringe pattern projections for three testing targets. Our experiments found that the triangular FPP provides the best imaging result and improves measurement quality. By properly selecting fringe patterns according to the target shape, the undesired harmonics of the measured surface can be eliminated from the phase maps. The triangular FPP is more suitable for 3D shape measurement with curved surfaces. Binary reflected gray-codes technique can be used in fields of topography, deflectometry, and interferometry for advanced measurement accuracies. We believe that further improvement is needed to achieve superior quality 3D shape measurements. Future works will be focused on developing an algorithm to generate optimal binary gray-codes fringe patterns for complex 3D shape measurements. The integration of the FPP system will be examined for fast measurement and the imaging time will be reduced to microsecond scale.

\section{ACKNOWLEDGMENT}

The authors would like to thank the Ministry of Science and Technology (MOST) of Taiwan and Yen Tjing Ling Medical Foundation to sponsor this study. The grant numbers are MOST 108-2221-E-010-012-MY3, MOST 109-2923-E-010001-MY2, and CI-109-24. 


\section{REFERENCES}

[1] Berryman, F., Pynsent, P., Fairbank, J., Disney, S. (2008). A new system for measuring three-dimensional back shape in scoliosis. European Spine Journal, 17, 663-673.

[2] Garnier, C., Pastor, M., Eyma, F., Lorrain, B. (2011). The detection of aeronautical defects in situ on composite structures using non destructive testing. Composite Structures, 93, 1328-1336.

[3] Kulkarni, R., Rastogi, P. (2016). Optical measurement techniques - a push for digitization. Optics and Lasers in Engineering, 87, 1-17.

[4] Servin, M., Quiroga, J., Padilla, J. (2014). Fringe Pattern Analysis for Optical Metrology: Theory, Algorithms, and Applications. Weinheim: Wiley-VCH.

[5] Salas, L., Luna, E., Salinas, J., Garcia, V., Servin, M. (2003). Profilometry by fringe projection. Optical Engineering, 42, 3307-3314.

[6] Chen, L., Quan, C., Tay, C., Fu, Y. (2005). Shape measurement using one frame projected sawtooth fringe pattern. Optics Communications, 246, 275-284.

[7] Wang, Z., Du, H., Bi, H. (2006). Out-of-plane shape determination in generalized fringe projection profilometry. Optics Express, 14, 12122-12133.

[8] Zhang, S., Yau, S. (2007). Generic nonsinusoidal phase error correction for three-dimensional shape measurement using a digital video projector. Applied Optics, 46, 36-43.

[9] Du, H., Wang, Z. (2007). Three-dimensional shape measurement with an arbitrarily arranged fringe projection profilometry system. Optics Letters, 32, 2438-2440.

[10] Pan, B., Kemao, Q., Huang, L., Asundi, A. (2009). Phase error analysis and compensation for nonsinusoidal waveforms in phase-shifting digital fringe projection profilometry. Optics Letters, 34, 416418.

[11] Zhang, S., Van der Weide, D., Oliver, J. (2010). Superfast phase-shifting method for 3-D shape measurement. Optics Express, 18, 9684-9689.

[12] Ayubi, G., Martino, J., Alonso, J., Fernández, A., Flores, J., Ferrari, J. (2012). Color encoding of binary fringes for gamma correction in 3-D profiling. Optics Letters, 37, 1325-1327.

[13] Zuo, C., Chen, Q., Gu, G., Feng, S., Feng, F., Li, R., Shen, G. (2013). High-speed three-dimensional shape measurement for dynamic scenes using bi-frequency tripolar pulse-width-modulation fringe projection. Optics and Lasers in Engineering, 51, 953-960.

[14] Feng, S., Chen, Q., Zuo, C., Sun, J., Yu, S. (2014). High-speed real-time 3-D coordinates measurement based on fringe projection profilometry considering camera lens distortion. Optics Communications, 329, 44-56.

[15] Li, X., Zhang, Z., Yang, C. (2016). Reconstruction method for fringe projection profilometry based on light beams. Applied Optics, 55, 9895-9906.
[16] Liu, C., Yen, T. (2016). Digital multi-step phaseshifting profilometry for three-dimensional ballscrew surface imaging. Optics \& Laser Technology, 79, 115123.

[17] Feng, S., Chen, Q., Zuo, C., Tao, T., Hu, Y., Asundi, A. (2017). Motion-oriented high speed 3-D measurements by binocular fringe projection using binary aperiodic patterns. Optics Express, 25, 540-559.

[18] Zhao, H., Xu, Y., Jiang, H., Li, X. (2018). 3D shape measurement in the presence of strong interreflections by epipolar imaging and regional fringe projection. Optics Express, 26, 7177-7131.

[19] Flores, J., Ayubi, G., Martino, J., Castillo, O., Ferrari, J. (2018). 3D-shape of objects with straight line-motion by simultaneous projection of color coded patterns. Optics Communications, 414, 185-190.

[20] Huang, T., Li, X., Fu, X., Zhang, C., Duan, F., Jiang, J. (2019). Arbitrary phase shifting method for fiber-optic fringe projection profilometry based on temporal sinusoidal phase modulation. Optics and Lasers in Engineering, 121, 300-306.

[21] Zhang, C., Zhao, H., Qiao, J., Zhou, C., Zhang, L., Hu, G., Geng, H. (2019). Three-dimensional measurement based on optimized circular fringe projection technique. Optics Express, 27, 2465-2477.

[22] Gai, S., Da, F., Tang, M. (2019). A flexible multi-view calibration and 3D measurement method based on digital fringe projection. Measurement Science and Technology, 30, 025203.

[23] Kemao, Q. (2004). Windowed Fourier transform for fringe pattern analysis. Applied Optics, 43, 2695-2702.

[24] Wang, H., Kemao, Q. (2009). Frequency guided methods for demodulation of a single fringe pattern. Optics Express, 17, 15118-15127.

[25] Kai, L., Kemao, Q. (2010). Fast frequency-guided sequential demodulation of a single fringe pattern. Optics Letters, 35, 3718-3720.

[26] Liu, K., Wang, Y., Lau, D., Hao, Q., Hassebrook, L. (2010). Dual-frequency pattern scheme for high-speed 3-D shape measurement. Optics Express, 18, 52295244.

[27] Ma, S., Quan, C., Zhu, R., Chen, L., Li, B., Tay, C. (2012). A fast and accurate gamma correction based on Fourier spectrum analysis for digital fringe projection profilometry. Optics Communications, 285, 533-538.

[28] Padilla, M., Servin, M., Garnica, G. (2017). Profilometry with digital fringe-projection at the spatial and temporal Nyquist frequencies. Optics Express, 25, 22292-22302.

[29] Qiao, N., Quan, C. (2018). Dual-frequency fringe projection for $3 \mathrm{D}$ shape measurement based on correction of gamma nonlinearity. Optics \& Laser Technology, 106, 378-384.

[30] Zhang, H., Zhao, H., Zhao, Z., Zhuang, Y., Fan, C. (2019). Two-frame fringe pattern phase demodulation using Gram-Schmidt orthonormalization with least squares method. Optics Express, 27, 10495-10508. 
[31] Singh, V., Tayal, S., Mehta, D. (2019). Single shot fringe projection profilometry using tunable frequency Fresnel biprism interferometer for large range of measurement. Optics Communications, 451, 104-110.

[32] Jia, P., Kofman, J., English, C. (2007). Multiple-step triangular-pattern phase shifting and the influence of number of steps and pitch on measurement accuracy. Applied Optics, 46, 3253-3262.

[33] Yang, Z., Wu, K., Xi, J., Yu, Y. (2014). Intensity ratio approach for 3D profile measurement based on projection of triangular patterns. Applied Optics, 53, 200-207.

[34] Herráez, M., Burton, D., Lalor, M., Gdeisat, M. (2002). Fast two-dimensional phase-unwrapping algorithm based on sorting by reliability following a noncontinuous path. Applied Optics, 41, 7437-7444.
[35] Zuo, C., Chen, Q., Feng, S., Feng, F., Gu, G., Sui, X (2012). Optimized pulse width modulation pattern strategy for three-dimensional profilometry with projector defocusing. Applied Optics, 51, 4477-4490.

[36] Xu, Y., Ekstrand, L., Dai, J., Zhang, S. (2011). Phase error compensation for three-dimensional shape measurement with projector defocusing. Applied Optics, 50, 2572-2581.

Received December 13, 2019 Accepted January 30, 2020 